\title{
Using Herschel and Planck observations to delineate the role of magnetic fields in molecular cloud structure (Corrigendum)
}

\author{
Juan D. Soler
}

\begin{abstract}
Max Planck Institute for Astronomy, Königstuhl 17, 69117, Heidelberg, Germany
\end{abstract} e-mail: soler@mpia.de

A\&A, 629, A96 (2019), https: //doi .org/10 . 1051/0004-6361/201935779

Key words. ISM: general - ISM: magnetic fields - ISM: clouds - dust, extinction - stars: formation - errata, addenda

There was an error in our Eq. (3). The correct equation should be:

$V \equiv \frac{\sum_{k}^{N} w_{k} \cos \left(2\left(\phi_{k}-\phi_{0}\right)\right)}{\sum_{k}^{N} \sqrt{\left(w_{k}\right)^{2} / 2}}$. 